\title{
Confidential and efficient asset proof for bitcoin exchanges
}

\author{
MAYA MOHAN $^{1, *}$, M K KAVITHA DEVI ${ }^{2}$ and V JEEVAN PRAKASH ${ }^{3}$ \\ ${ }^{1}$ Department of Computer Science and Engineering, NSS College of Engineering, Palakkad 678008, India \\ ${ }^{2}$ Department of Computer Science and Engineering, Thiagarajar College of Engineering, Madurai 625015, India \\ ${ }^{3}$ Department of Mathematics, NSS College of Engineering, Palakkad 678008, India \\ e-mail: mayajeevan@gmail.com
}

MS received 9 February 2017; revised 5 July 2017; accepted 21 September 2017; published online 30 June 2018

\begin{abstract}
Technological advancements boost the business to play a crucial role in a country's economic success. Cryptography-based currencies, called as cryptocurrencies, are now leading the world's economy. With the increasing popularity of cryptocurrencies, cryptocurrency exchanges have emerged to support cryptocurrency-related services. Among cryptocurrencies, bitcoin takes the lead and it is widely accepted by the world community. Lots of security issues are arising day by day and the exchange should handle all of of them sensibly. It is necessary that the exchange should be solvent all the time in terms of its assets and liabilities for its survival. For this, periodic settlement of the accounts should be done using appropriate techniques. The information exchange needed for this should be concealed from the adversaries. Cryptography-based techniques with zero-knowledge protocols are suitable for this purpose. Maxwell's proof of liabilities is the first cryptography-based method to verify the user assets. It makes use of binary Merkle hash trees for representing the proof of liabilities. The root node reveals the total assets of the exchange, which will attract the adversaries to execute an attack. Later the Dagher et al scheme, a privacy preserving proof of asset for bitcoin exchanges, was proposed. The scheme works in an interactive manner that requires the collaboration of the exchange and the user. This paper proposes an efficient non-interactive proof of assets for bitcoin exchanges.
\end{abstract}

Keywords. Cryptocurrency; bitcoin exchange; zero-knowledge proof; commitment scheme; proof of assets; non-programmable random oracle model.

\section{Introduction}

In the trading history, money has taken different forms over time and the latest addition is the virtual currency [1,2]. Virtual currencies are similar to digital currencies, but have their own currency units. Cryptocurrencies are the youngest generation of virtual currencies that gained lots of attention from the economists. All over the world, around 280 cryptocurrencies exist today and bitcoin, Litecoin and Dogecoin are some of them. Cryptocurrencies heavily use cryptographic algorithms for their design. According to a survey conducted using Google drive, it is identified that bitcoin is the most trading cryptocurrency with a market capital around $\$ 40$ billion [3]. Ethereum, Ripple, Dash and Litecoin are other leading cryptocurrencies in the cryptocurrency market [4]. The potential to increase the payment efficiency with minimum transaction cost is the main success behind cryptocurrencies. With the increase in popularity of cryptocurrencies, cryptocurrency exchanges are introduced to support the currency-related services. According to the current statistics more than 50 large and

*For correspondence small scale cryptocurrency exchanges are functioning all over the world. Since bitcoin acquired the market capital, bitcoin exchanges are dominating over other cryptoexchanges.

The exchange holds the personal credentials and the account information of each user. The exchange creates a wallet for each user to perform the bitcoin trading. While selling the bitcoin through the exchange, clients have to transfer the bitcoins to the wallet allocated to them in the exchange. It is the user's responsibility to choose the proper exchange, since the private key of the wallet is with the exchange. The transactions performed by the user through exchanges are added to the blockchain as exchange-initiated transactions, which provide total anonymity for the users. The exchange's service includes trading of cryptocurrencies, exchange of cryptocurrencies with fiat currencies, merchant services, mining, etc. Public key cryptosystems are used to provide security for bitcoins and also for the communication between the exchange and the customers.

Various socio-economic factors determine the survivability of an exchange such as transaction volume, solvency, attack survivability and market catch up. The 
exchange survival time can be estimated using either the Cox proportional hazard model or the best fix Cox model. Solvency of an exchange is based on its assets and liabilities. If the exchange is solvent enough with respect to its assets and liabilities, it can easily solve the compliance and settlement issues. Using proof of solvency, the exchange can prove that it has enough reserves to meet the customer assets. The financial security can be assured with timely auditing of the assets and liabilities. The cryptographybased algorithms are helpful in proving the reserves, such as the one used in Kraken exchange. Proof of solvency helps the users and exchanges to convince each other about the assets and liabilities without revealing any private information. Maxwell proposed a method for proving the liability of an exchange using Merkle hash trees [5, 6]. User verification is also possible with this method. Hash-based Merkle tree represents the hashed credentials of the user and their balance. The total liability is summed up in the root node. The drawback with this approach is that it reveals the total liability, which is available in the root node. In 2015 a privacy preserving proof of solvency for bitcoin exchanges was introduced [7]. The method provides proof of assets and proof of liabilities by preserving the privacy of the user and the exchange. To build the proof the protocol uses commitment schemes and zero-knowledge proofs. It is necessary for the protocol to have an interaction between the user and the exchange to complete the proof.

In this paper an efficient privacy preserving non-interactive proof of assets (EPoA) for bitcoin exchanges is proposed. Non-interactive zero-knowledge (NIZK) proofs are a category of zero-knowledge proofs in which no communication is required between the prover and the verifier to compute the proof. The proposed scheme uses the Perdersen commitment scheme and sigma protocol for computing the proof. A common reference string (CRS) is required for the NIZK proofs and that should be shared between the prover and the verifier priorly. A simple scenario for interactive and non-interactive proofs is given, respectively, in figures 1 and 2 . Outline of the paper is as follows. In section 2, the preliminaries and definitions related to sigma protocol and zero-knowledge proofs are

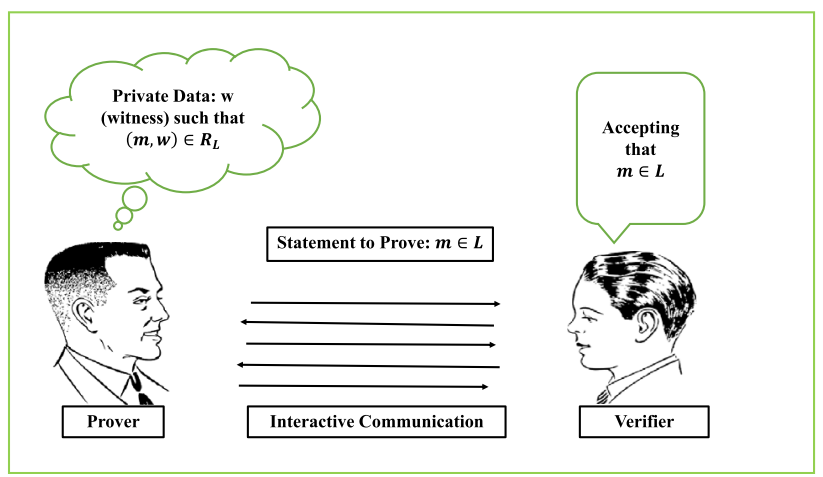

Figure 1. Interactive protocol.

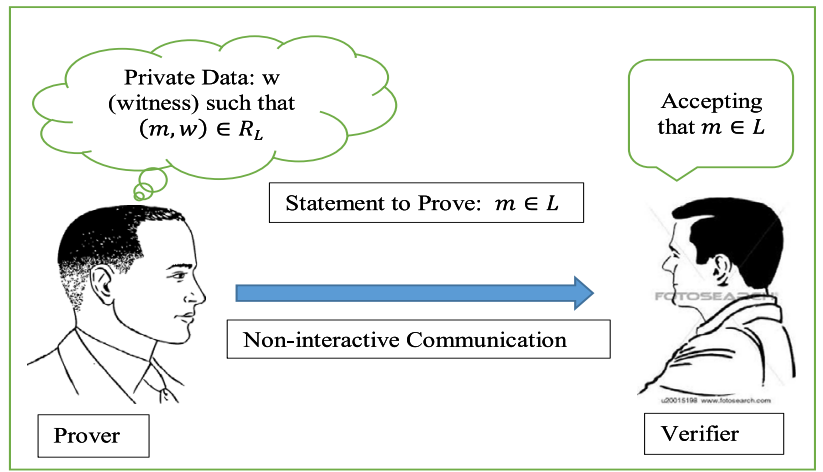

Figure 2. Non-interactive protocol.

discussed. Section 3 discusses about confidential and efficient proof of assets for bitcoin exchanges. In section 4 , the results and discussions are included and section 5 provides the conclusion.

\section{Preliminaries and definitions}

For building the proofs, the protocol make use of Pedersen commitments, zero-knowledge proofs and sigma protocols [8]. Commitment schemes allow the users to hide (commit) the message for a certain time and ensures that the message cannot be altered during the commitment process. Zeroknowledge protocols allow the prover to prove some facts to the verifier without revealing any additional information [9]. It is widely used in multiparty applications.

Definition 2.1 Sigma protocols $\left(\sum\right)$ are 3-round public coin honest verifier zero-knowledge (HVZK) protocols with a prover $P$ and a verifier $V$ to prove $m \in L$ [10]. It follows the following steps:

- on input $1^{k}, m$ and the witness $w, P$ computes the random value $a$ and sends it to $V$;

- on receiving $a, V$ sends a random challenge $c h \in Z_{q}$ with length $t$ to $P$;

- with the input $(m, a, c h, w), P$ computes $z$ and sends it to $V$;

- $V$ accepts or rejects based on ( $m, a, c h, z)$.

The prover and the verifier have access to a common shared string that is chosen in a random way, known as CRS in adaptive interactive zero-knowledge proofs [11]. The statements are considered as CRS functions in order to prove the properties of zero knowledge and soundness [12]. The soundness property is defined in the non-programmable random oracle model (NPRO). The definition for adaptive unbounded zero knowledge is given here.

Definition 2.2 A relation $R$ is defined in the language $L \in N P$ for any probabilistic polynomial time (PPT) 
algorithm (GenCRS, $P, V$ ) holding adaptive non-interactive unbounded zero-knowledge property if the following conditions are satisfied:

- Perfect completeness: $\rho \leftarrow_{R} \operatorname{GenCRS}\left(1^{k}\right) \forall(m, w) \in R$ $\operatorname{Prob}[V(m, \rho, P(m, w, \rho))=1]=1$.

- Adaptive Soundness defined in NPRO: For every PPT function $f$ and PPT $P^{*}$ (cheating prover)

$\operatorname{Prob}\left[V\left(\rho, f(\rho), P^{* F}(\rho)\right)=1\right] \leq v(k) \forall k \in Z_{q}$ where

$f:\{0,1\}^{k} \backslash L \leftarrow\{0,1\}^{p o l y(k)} v \leftarrow$ negligible function

$F:\{0,1\}^{k} \leftarrow\{0,1\}^{*}$ any random function

$\rho \leftarrow \operatorname{GenCRS}\left(1^{k}\right)$.

- Adaptive unbounded zero knowledge: For very PPT function $f$, there exists a PPT simulator Sim for the language $L \in N P$ and the relations $R$ such that for the random challenge $c h$ is defined as $f$ : $\{0,1\}^{k} \times\{0,1\}^{\text {poly }(k)} \cap R \leftarrow\{0,1\}^{\text {poly }(k)}$

and for every PPT $V^{*}$ (cheating verifier) the difference in probability is negligible.

$\operatorname{Pr}\left[V^{*}\left(\operatorname{Real}_{f}\left(P^{f}(k, p)\right)\right)=1\right]$

$-\operatorname{Pr}\left[V^{*}\left(\operatorname{Sim}_{f}(k, p)\right)=1\right] \leq v(k)$.

\section{Confidential and efficient asset proofs}

The exchange should have proper mechanisms to hide its customer credentials as well as the total assets. Unlinkability of bitcoin exchanges with their bitcoin addresses ensures complete privacy. In Provisions [7], interaction is required between the exchange and the user to complete the proof. This will limit the exchange to prove its assets individually. In EPoA, this is accomplished using the NPRO model. Different non-interactive protocols are considered for obtaining efficient asset proofs for bitcoin exchanges. The initial set-ups and Pedersen commitment calculations are similar for all the schemes. The exchange collects all the public keys from the bitcoin blockchain and creates the anonymous set $P K$.

$$
P K=\left\{Y_{1}, Y_{2}, Y_{3}, \ldots Y_{n}\right\} \text { where } n \in Z_{q}
$$

The proof size of EPoA is directly proportional to the size of PK. The exchange maintains a set of secret keys $S K=$ $\left\{x_{1}, x_{2}, \ldots x_{n}\right\}$ such that the public key $Y_{i}=g^{x_{i}}$ where $1 \leq i \leq n$ and $g$ is the generator of group $G$. Each exchange gathers the public key available in the blockchain and sets a boolean value $o_{i} \in[0,1]$ that corresponds to the account the public key holds. The $o_{i}$ value is set to one for the accounts the exchange knows; else the private key is set to zero. The balance associated with each account is a positive value denoted as $B\left(Y_{i}\right)$. The commitment for the total asset is computed using Eq. (1):

$$
T A=\sum_{i=1}^{n} o_{i} \cdot B\left(Y_{i}\right) \forall i \in[1, n] .
$$

Compute the DH tuple for $B\left(Y_{i}\right)$ to perform the proof computation as given in Eq. (2):

$$
B_{i}=g^{B\left(Y_{i}\right)} \forall i \in[1, n] .
$$

The exchange calculates the commitment values for $o_{i} B\left(Y_{i}\right), o_{i}$ and $x_{i}$ as given in Eqs. (3)-(6):

$$
\begin{gathered}
C_{i}=B_{i}^{o_{i}} h^{r_{i}}, \\
D_{i}=Y_{i}^{o_{i}} h^{k_{i},}, \\
D_{i}=g^{x_{i} \cdot o_{i}} h^{k_{i}} .
\end{gathered}
$$

Whenever exchange knows the private key, then $o_{i}=1$; else $o_{i}=0$; then the commitment can be written as

$$
D_{i}=g^{x_{i}} h^{k_{i}}
$$

where $g$ and $h$ are the generators of the group $G$ and $r_{i}, k_{i} \in \in_{R} Z_{q}, i \in[1, n]$. The commitment for the total asset of the exchange $C_{T A}$ is obtained by homomorphically adding the individual commitment for each balance as shown in Eq. (7). The Pedersen commitment is used for this purpose, which exhibits homomorphic property [13]:

$$
C_{T A}=\prod_{i=1}^{n} C_{i}=\prod_{i=1}^{n} B_{i}^{o_{i}} h^{r_{i}}
$$

The exchange has to prove in zero knowledge that the commitment for the total asset is computationally correct and the private values $o_{i}, r_{i}, k_{i}$ and $x_{i}$ are known to the exchange. This is proved using NIZK protocol. A privacy preserving non-interactive proof of asset scheme is realized using hybrid commitment scheme in the NPRO model $[14,15]$. For the analysis purpose the scheme is explained as follows.

Public values: $G, g, h, B_{i}, C_{i}, D_{i}, Y_{i}$.

CRS generation: $\rho \leftarrow\left(1^{k}\right), k \in_{R} Z_{q}$.

Compute $u$ and $v, u=g^{\rho}$ and $v=h^{\rho}$ are equivocal and $u=g^{\rho_{1}}$ and $v=h^{\rho_{2}}$ are perfectly binding, where $g$ and $h$ are generators of the multiplicative group $G$;

$s \leftarrow$ secret key for the hash function.

The CRS generated is $(\rho, s, u, v)$.

Prover side

For $i=1$ to $n^{\prime}$, where $n^{\prime}$ is the number of public keys in $P K$, exchange picks the values $\alpha, \beta, \gamma, \delta$ and $t \in Z_{q}$.

1 . The $a$ values for the sigma protocol are

$$
\begin{aligned}
& a_{1 i}=B_{i}{ }^{\alpha} h^{\beta}, \\
& a_{2 i}=Y_{i}^{\alpha} h^{\gamma}, \\
& a_{3 i}=g^{\delta} h^{\gamma} .
\end{aligned}
$$

2. The commitment for $a$ is 


$$
\begin{aligned}
& \operatorname{Com}_{i}=\left(\operatorname{Com}_{1 i}, \operatorname{Com}_{2 i}, \operatorname{Com}_{3 i}, \operatorname{Com}_{4 i}, \operatorname{Com}_{5 i}, \operatorname{Com}_{6 i}\right) \text {, } \\
& \operatorname{Com}_{1 i}=g^{t} / u^{a_{1 i}}, \quad \operatorname{Com}_{2 i}=h^{t} / v^{a_{1 i}}, \quad \operatorname{Com}_{3 i}=g^{t} / u^{a_{2 i}} \text {, } \\
& \operatorname{Com}_{4 i}=h^{t} / v^{a_{2 i}}, \quad \operatorname{Com}_{5 i}=g^{t} / u^{a_{3 i}}, \quad \operatorname{Com}_{6 i}=h^{t} / v^{a_{3 i}} \text {. }
\end{aligned}
$$

3. Compute the hash of the committed values $\operatorname{Com}_{i}$ and $C_{T A}$ :

$$
c h_{i}=H_{s}\left(\operatorname{Com}_{i}, C_{T A}\right)
$$

4. The $Z$ values are computed as follows:

$$
\begin{aligned}
& z_{1 i}=\alpha+\left(c h_{i} o_{i}\right), \\
& z_{2 i}=\beta+\left(c h_{i} r_{i}\right), \\
& z_{3 i}=\gamma+\left(c h_{i} k_{i}\right), \\
& z_{4 i}=\delta+\left(c h_{i} x_{i}\right) .
\end{aligned}
$$

5. Publish the values $\left(a_{1 i}, a_{2 i}, a_{3 i}, z_{1 i}, z_{2 i}, z_{3 i}, z_{4 i}\right.$, $\left.\operatorname{Com}_{i}, C_{T A}\right)$. The exchange can decommit the value at a later time by privately sending the random value $t$.

Verifier side

Verifier accepts the proof if

$$
\begin{aligned}
& B_{i}^{z_{1 i}} h^{z_{2 i}}=C_{i}^{c h_{i}} a_{1 i}, \\
& Y_{i}^{z_{1 i}} h^{z_{3 i}}=D_{i}^{c h_{i}} a_{2 i}, \\
& g^{z_{4 i}} h^{z_{3 i}}=D_{i}^{c h_{i}} a_{3 i} .
\end{aligned}
$$

Exchange's total asset commitment is computed as

$$
C_{T A}=\prod_{i=1}^{n} C_{i} .
$$

This approach uses the dual mode commitment scheme [16] for computing the non-interactive proofs. The scheme satisfies the completeness, adaptive soundness and zeroknowledge properties in the non-programmable random oracle with CRS.

The limitation of this scheme is the extra computation required to run the sigma protocol to complete the NIZK proof. The hybrid commitment scheme using sigma protocol $\sum=(P, V)$ for the language $L$ with commitment 'com' exhibits an efficiency loss due to the message space of the hybrid commitment scheme (dual mode commitment scheme). In a fixed CRS, the maximum length of a message committed to a single 'com' is limited to the challenge length $l_{L^{\prime}}$ for the membership hard language $L^{\prime}$. This will demand multiple executions of com if ' $a$ ' of $\sum_{L}$ is much longer than the size of $l_{L^{\prime}}$. This will lead to a huge efficiency loss.

The FS transform realized in the random oracle model produces the NIZK proofs with less computation compared with Lindell's transform. There is a remarkable difference in generality and efficiency of Lindell's transform compared with FS transform. The asset proof can be realized using FS transform as follows. The commitment calculation can be simply avoided to execute the FS transform. FS transform does not require the CRS set-up.

Public values: $G, g, h, B_{i}, C_{i}, D_{i}, Y_{i}$

$g$ and $h$ are generators of the multiplicative group $G$

$s \leftarrow$ secret key for the hash function

Prover side

For $i=1$ to $n^{\prime}$, where $n^{\prime}$ is the number of public keys in $P K$, exchange picks the values $\alpha, \beta, \gamma, \delta$ and $t \in Z_{q}$.

1. The $a$ values for the sigma protocol are

$$
\begin{aligned}
& a_{1 i}=B_{i}{ }^{\alpha} h^{\beta}, \\
& a_{2 i}=Y_{i}^{\alpha} h^{\gamma}, \\
& a_{3 i}=g^{\delta} h^{\gamma} .
\end{aligned}
$$

2. Compute the hash of the $a$ values:

$$
\operatorname{hash}_{i}=H_{s}\left(a_{1 i}, a_{2 i}, a_{3 i}\right) .
$$

3. The $Z$ values are computed as follows:

$$
\begin{aligned}
& z_{1 i}=\alpha+\left(\operatorname{hash}_{i} o_{i}\right), \\
& z_{2 i}=\beta+\left(\text { hash }_{i} r_{i}\right), \\
& z_{3 i}=\gamma+\left(\operatorname{hash}_{i} k_{i}\right), \\
& \left.z_{4 i}=\delta+\operatorname{hash}_{i} x_{i}\right) .
\end{aligned}
$$

4. Publish the values $\left(a_{1 i}, a_{2 i}, a_{3 i}, z_{1 i}, z_{2 i}, z_{3 i}, z_{4 i}, C_{T A}\right)$. The exchange can decommit the value at a later time by privately sending the random value $t$.

Verifier side

Verifier accepts the proof if

$$
\begin{aligned}
& B_{i}^{z_{1 i}} h^{z_{2 i}}=C_{i}^{\text {hash }_{i}} a_{1 i}, \\
& Y_{i}^{z_{1 i}} h^{z_{3 i}}=D_{i}^{\text {hash }_{i}} a_{2 i}, \\
& g^{z_{4 i}} h^{z_{3 i}}=D_{i}^{\text {hash }_{i}} a_{3 i} .
\end{aligned}
$$

Exchange's total asset commitment is computed as

$$
C_{T A}=\prod_{i=1}^{n} C_{i} .
$$

In the FS transform all entities have access to the oracle $O$, which on input $x$ always outputs the random value $y$. The simulator has complete control over the oracle and it can program the oracle. The simulator's query output is completely indistinguishable from the real query output. FS transform satisfies the completeness, soundness and zeroknowledge properties. While considering the FS transform, in the general scenario and some specific cases the random oracle methodology is proved to be unsound. Moreover the constructions proved to be secure in the random oracle model are identified as insecure for any concrete instantiation of the hash function used.

To improve the efficiency of the proof of assets a NIZK proof in the NPRO model that provides optimal soundness 
is used [17]. According to the scheme, if the statement $m \in L$ then for ' $a$ ' there will be at most one challenge that can be computed correctly. The sigma protocol for the language $L$ and the membership hard language $L^{\prime}$ are 3round public coin HVZK proofs having optimal soundness. Multiple executions of com based on the challenge length in Lindell Transform is omitted in the Ciampi et al transform. The Ciampi et al transform does not require the perfect HVZK and special HVZK properties for the $\sum$ protocol used. However, the scheme demands generality and it is not necessary that HVZK proofs should be witness indistinguishable.

The Ciampi et al transform makes use of OR proofs. In this scheme the witness is given to the simulator. This security problem is eliminated using OR construction of 3-round perfect HVZK proofs. The prover $P$ has to prove in NIZK that any single statement holds true, that is either $m \in L$ or $\rho \in L^{\prime}$. The OR construction gives WI only to two instances of the same language. It uses the generalized form of the proof that helps in OR composition of dissimilar protocols for nonidentical languages with computational HVZK.

Definition 3.1 If a 3-round public coin protocol $\pi=$ $(P, V)$ for a language $L$ is witness indistinguishable for a relation $R$, then for all cheating verifier $V^{*}$ there exists a negligible function $v$ such that $\forall m, w, w^{\prime}$ the relations $(m, w) \in R$ and $\left(m, w^{\prime}\right) \in R$ hold and,

$$
\begin{aligned}
& \left|\operatorname{Prob}\left[\left\langle P(w), V^{*}\right\rangle(m)=1\right]-\operatorname{Prob}\left[\left\langle P\left(w^{\prime}\right), V^{*}\right\rangle(m)=1\right]\right| \\
& \quad \leq v(|m|) .
\end{aligned}
$$

The algorithm exhibits perfect HVZK when $v(|m|)=0$. The challenge length extension is possible with a 3-round public coin protocol holding perfect HVZK and optimal soundness. The challenge length amplification is done by multiplying the parallel instances of 3-round public coin protocol with the length of the challenge. It is possible to achieve the same perfect HVZK and optimal soundness for

Table 1. OR construction for $L \vee L$.

\begin{tabular}{ll}
\hline Prover & \\
\hline 1 & $a_{s}=P_{L}\left(m_{s}, w\right)$ \\
2 & $\left(a_{1-s}, c h_{1-s}, z_{1-s}\right) \leftarrow \operatorname{Sim}_{L}\left(z_{1-s}\right)$ \\
3 & The ' $a$ ' values $\left(a_{0}, a_{1}\right)$ are send to $V_{L \vee L}$ \\
5 & Compute $c h_{s}=c h \oplus c h_{1-s}$ \\
6 & Compute $z_{s} \leftarrow P_{L}\left(m_{s}, w, a_{s}, c h_{s}\right)$ \\
7 & Send the pairs $\left(c h_{0}, c h_{1}\right)\left(z_{0}, z_{1}\right)$ to $V_{L \vee L}$ \\
\hline Verifier & \\
\hline 4 & The random challenge \\
& $c h \leftarrow\{0,1\}^{l}$ send to $P_{L \vee L}$ \\
8 & Accept if $c h=c h_{0} \oplus c h_{1}$ \\
& $V_{L}\left(m_{0}, a_{0}, c h_{0}, z_{0}\right)=0$ and \\
& $V_{L}\left(m_{1}, a_{1}, c h_{1}, z_{1}\right)=1$ \\
\hline
\end{tabular}

the instances of 3-round public coin protocol with lesser challenge length. Random pad bits are appended with the challenge to acquire the desired length.

According to the Cramer et al scheme [18], using a 3round public-coin HVZK proof system $\pi_{L}$ for an NP language $L$, construct a 3-round public-coin HVZK proof system $\pi_{L \vee L}$ for the NP language $L \vee L$ such that

$$
L \vee L=\left(m_{0}, m_{1}\right): m_{0} \in L \vee m_{1} \in L .
$$

The 3-round public coin protocol is HVZK for the language $L \vee L$ with the instances $m_{0}, m_{1} \in_{R} L$ given in table 1 . $\operatorname{Sim}_{L}$ is the simulator for $\pi_{L}$ and the random bit $s \in[0,1]$. The relation $R_{L \vee L}$ for the NP language $L \vee L$ in 3-round public coin HVZK proof system is $R_{L \vee L}=\left(\left(m_{0}, m_{1}\right), w\right)$ such that

$$
\left(\left(m_{0}, w\right) \in R_{L} \wedge m_{1} \in L\right) \vee\left(\left(m_{1}, w\right) \in R_{L} \wedge m_{0} \in L\right) .
$$

The same construction is applied in the Ciampi et al transform to prove the relation for the NP language $L \vee L^{\prime}$ in 3-round public coin HVZK proof system. For the NP language, $L \vee L^{\prime}=\left(m_{0}, m_{1}\right): m_{0} \in L \vee m_{1} \in L^{\prime}$ the relation $R_{L} \vee L^{\prime}$ is $R_{L \vee L^{\prime}}=\left(\left(m_{0}, m_{1}\right), w\right)$ such that

$$
\left(\left(m_{0}, w\right) \in R_{L} \wedge m_{1} \in L^{\prime}\right) \vee\left(\left(m_{1}, w\right) \in R_{L^{\prime}} \wedge m_{0} \in L\right) .
$$

If the 3-round public coin protocols $\pi_{L}$ and $\pi_{L^{\prime}}$ for the languages $L$ and $L^{\prime}$ are perfect witness indistinguishable then $\pi_{L \vee L^{\prime}}$ is perfect WI for the polynomial relation $R_{L \vee L^{\prime}}$.

In the Ciampi et al transform, commitment schemes are not used. The $\rho$ generated from the CRS is used in the computation of ' $a$ ' value in the non-interactive version of the sigma protocol. This is the highlight of the transform compared with the FS transform. The scheme satisfies completeness, optimal soundness and zero knowledge in the NPRO model using CRS. Using the Ciampi et al transform the proof of assets protocol(EPoA) can be proved in a noninteractive way in the NPRO model using CRS as follows.

Public values: $G, g, h, B_{i}, C_{i}, D_{i}, Y_{i}$

CRS generation: $\left(\rho_{1}, \rho_{2}\right) \leftarrow\left(1^{k}\right), k \in_{R} Z_{q}$ $g$ and $h$ are generators of the multiplicative group $G$ $s \leftarrow$ is the secret key for the hash function. The CRS generated is $(\rho, s, u, v)$.

Prover side

For $i=1$ to $n^{\prime}$, where $n^{\prime}$ is the number of public keys in $P K$, exchange picks the values $\alpha, \beta \in Z_{q}$.

1. The $a$ values for the sigma protocol are

$$
\begin{aligned}
& a_{1 i}=B_{i}{ }^{\rho_{1}} h^{\alpha}, \\
& a_{2 i}=Y_{i}{ }^{\rho_{1}} h^{\rho_{2}}, \\
& a_{3 i}=g^{\beta} h^{\rho_{2}} .
\end{aligned}
$$

2. Compute the hash of $a$ values $C_{T A}$ :

$$
h c_{i}=H_{s}\left(C_{T A}, a_{1 i}, a_{2 i}, a_{3 i}\right) .
$$

3. The $Z$ values are computed as follows: 


$$
\begin{gathered}
z_{1 i}=\rho_{1}+\left(h c_{i} o_{i}\right), \\
z_{2 i}=\alpha+\left(h c_{i} r_{i}\right), \\
z_{3 i}=\rho_{2}+\left(h c_{i} k_{i}\right), \\
z_{4 i}=\beta+\left(h c_{i} x_{i}\right) .
\end{gathered}
$$

4. Publish the values $\left(a_{1 i}, a_{2 i}, a_{3 i}, z_{1 i}, z_{2 i}, z_{3 i}, z_{4 i}, C_{T A}\right)$.

Verifier side

Verifier accepts the proof if

$$
\begin{aligned}
& B_{i}^{z_{1 i}} h^{z_{2 i}}=C_{i}^{h c_{i}} a_{1 i}, \\
& Y_{i}^{z_{11}} h^{z_{3 i}}=D_{i}^{h c_{i}} a_{2 i}, \\
& g^{z_{4 i}} h^{z_{3 i}}=D_{i}^{h c_{i}} a_{3 i} .
\end{aligned}
$$

Exchange's total asset commitment is computed as

$$
C_{T A}=\prod_{i=1}^{n} C_{i}
$$

According to Garay et al [19] if $\pi_{L}=\left(P_{L}, V_{L}\right)$ is a 3-round public coin witness indistinguishable proof system for the polynomial relation $R_{L}$ then the protocol $\pi=(P, V)$ is adaptive witness indistinguishable for $R_{L}$ in CRS model. The soundness can be proved using a method similar to that adopted in the Lindell scheme, considering the fact that for the random function $f n$ holding a large target set, the polynomial relation $R=(m, f n(m))$ is ambiguous in the NPRO model [20]. A polynomial relation $R$ is said to be ambiguous if, querying to the random oracle $O$, it is infeasible to find $m$ such that $(m, O(m)) \in R$. Consider $\pi_{L \vee L^{\prime}}=\left(P_{L \vee L^{\prime}}, V_{L \vee L^{\prime}}\right)$ to be a 3-round HVZK proof system holding optimal soundness for the polynomial relation

$$
\hat{R}_{L \vee L^{\prime}}=((m, \rho), w)
$$

such that

$$
\left((m, w) \in R_{L} \wedge \rho \in \hat{L^{\prime}}\right) \vee\left((\rho, w) \in R_{L^{\prime}} \wedge m \in \hat{L}\right)
$$

is witness indistinguishable for the polynomial relation

$$
R_{L \vee L^{\prime}}=((m, \rho), w)
$$

such that

$$
\left((m, w) \in R_{L} \wedge \rho \in L^{\prime}\right) \vee\left((\rho, w) \in R_{L^{\prime}} \wedge m \in L\right) .
$$

Then $\pi$ zero-knowledge property holds for the relation $R_{L}$ in the CRS model.

\section{Results and discussion}

In order to realize the advantage of non-interactiveness, a computationally efficient protocol is designed in the non-

\begin{tabular}{|c|c|c|c|c|c|c|c|c|}
\hline \multirow[t]{3}{*}{ Schemes } & \multicolumn{4}{|c|}{$|P|=\mathbf{1 0 2 4}$} & \multicolumn{4}{|c|}{$|P|=\mathbf{2 0 4 8}$} \\
\hline & \multicolumn{2}{|c|}{$P$} & \multicolumn{2}{|c|}{$V$} & \multicolumn{2}{|c|}{$P$} & \multicolumn{2}{|c|}{ V } \\
\hline & $\mathrm{com}$ & $\Sigma$ & com & $\Sigma$ & com & $\Sigma$ & com & $\Sigma$ \\
\hline $\begin{array}{l}\text { Lindell } \\
\text { Transform }\end{array}$ & 12 & 2 & 12 & 4 & 20 & 2 & 20 & 4 \\
\hline $\begin{array}{l}\text { Ciampi et al } \\
\text { Transform }\end{array}$ & & 2 & 4 & 4 & 4 & 2 & 4 & 4 \\
\hline $\begin{array}{l}\text { Fiat Shamir } \\
\text { Transform }\end{array}$ & - & 2 & - & 4 & - & 2 & - & 4 \\
\hline
\end{tabular}
programmable random oracle (NPRO) model. As discussed in the previous section, FS transform satisfies soundness property only in the RO model. To overcome the limitations, a non-interactive proof of assets is designed in the NPRO model using Pedersen commitments. Parallel execution of the components are possible with EPoA because of the linearity in the structure. The address set of the protocol is perfectly separable with unique independent component for each address belonging to the address set. Apart from the hash computation, the time complexity is the same for the Dagher et al scheme and EPoA. Proof of

Figure 3. Transforms comparison.

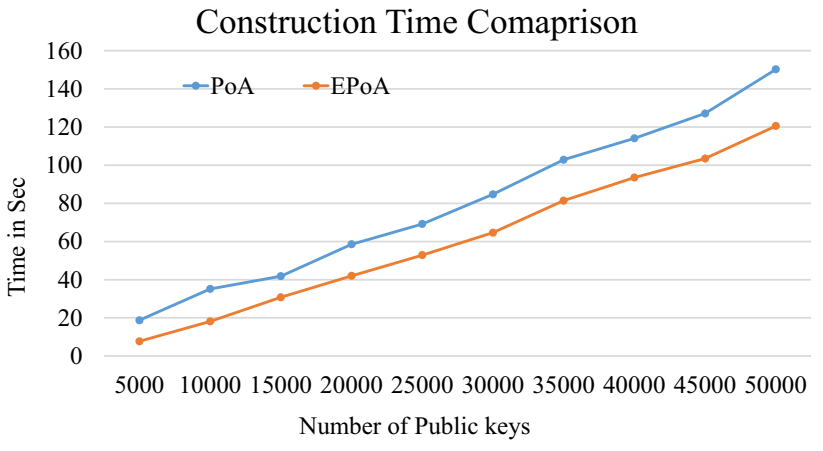

Figure 4. Proof construction time comparison.

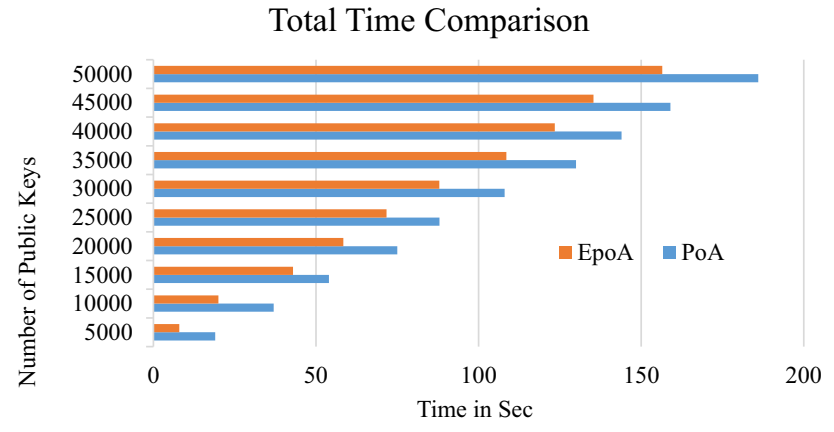

Figure 5. Total time comparison. 


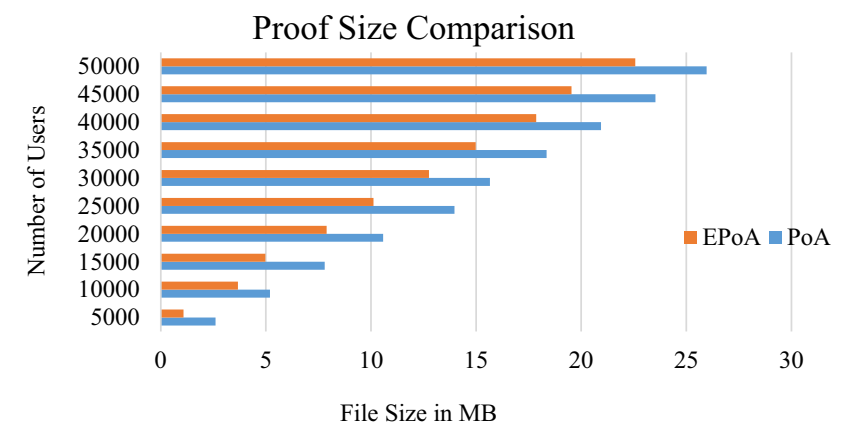

Figure 6. Proof size comparison.

assets with Lindell tranform requires a total of six component exponentiations for the final commitment calculation. The hash function provides integrity for the total commitment as well as the $a$ values, which is lacking in the Dangher et al scheme. A computational analysis of the three schemes carried out by Ciampi et al is given in figure 3. EPoA protocol does not support Multisig addresses. The proof size can be further reduced using zk-SNARKs [21]. Hardware Security Modules (HSM) [22]-enabled exchanges make use of ECDSA signatures that do not support the raw multiplications performed in EPoA. These are some of the limitations of EPoA, which should be noted by the research community. The protocol is implemented and tested in Java 1.8 with the support of standard java library Bouncy Castle. The performance analysis is carried out using 50000 public keys. The protocol can support an even larger anonymity set. The computation time and the proof size of the protocol EPoA compared with the proof of assets realization using Lindell scheme (PoA) are given in figures 3, 4, 5 and 6 . The proof computation time includes the construction and verification time. The outcome shows that the proposed protocol gives better performance in proof computation time and proof size compared with PoA protocol.

\section{Conclusion}

Bitcoin has become the potential candidate among virtual currencies. The wide usage of bitcoin has increased its market capitalization of 40 billion dollars. Nowadays online shoppings are carried out using bitcoin. This demands the existence of exchanges. It is adequate to have measures to provide privacy for the information stored in the exchanges. The EPoA protocol enables the exchange to prove its assets in zero knowledge in a non-interactive manner to its customers. The Dangher et al scheme is made non-interactive using the NPRO model using the Cimapi et al scheme. We have seen the drawbacks in using FS transform in the random oracle model as well as Lindell scheme in the NPRO model. The EPoA protocol with NPRO helps in publishing the proof efficiently without any verifier interaction. The privacy of the exchange is well preserved without much computation. The method also provides integrity for the data using hash functions. The computation analysis of the three schemes is well explained and the major issues are identified.

\section{References}

[1] Belenkiy M 2011 E-cash. In: Rosenberg B (Ed.) Handbook of financial cryptography and security. London: CRC, pp. 4-48

[2] Parhonyi R 2011 Micropayment systems. In: Rosenberg B (Ed.) Handbook of financial cryptography and security. London: CRC, pp. 161-183

[3] Nakamoto S 2008 Bitcoin: a peer-to-peer electronic cash system, vol. 1. New York: BN Publishing, p. 2012

[4] Okamoto T and Ohta K 1992 Universal electronic cash. In: Proceedings of CRYPTO 91, LNCS, vol. 576, pp. 324337

[5] Wilcox Z 2014 Proving your bitcoin reserves. https://iwilcox. me.uk/2014/proving-bitcoin-reserves. Accessed 27 Feb 2014

[6] Merkle R C 1979 Secrecy, authentication and public key systems. Ph.D. thesis, Stanford University

[7] Dagher G G, Bunz B, Bonneau J, Clark J and Boneh D 2015 Provisions: privacy preserving proofs of solvency for bitcoin exchanges. In: Proceedings of ACM CCS 2015, pp. 720-731

[8] Pedersen T P 1991 Non-interactive and information-theoretic secure verifiable secret sharing. In: Proceedings of Advances in Cryptology-CRYPTO 91, pp. 129-140

[9] Damgard I 1989 On the existence of bit commitments schemes and zero knowledge proofs. In: Proceedings of CRYPTO'89, LNCS 435. Santa Barbara, USA: Springer, pp. $17-27$

[10] Damgard I 2010 On $\sum$ protocols. http://www.daimi.au.dk/ $\sim$ ivan/sigma.pdf. Accessed 2 July 2010

[11] De Santis A, Micali S and Persiano G 1987 Non-interactive zero knowledge proof systems. In: Proceedings of Advances in Cryptology-CRYPT'87: A Conference on the Theory and Applications of Cryptographic Techniques, Santa Barbara, California, USA, pp. 52-72

[12] Chaidos P and Groth J 2015 Making sigma protocols noninteractive without random oracles. In: Proceedings of $P K C$ 2003, LNCS 9020. Florida: Springer, pp. 650-670

[13] Ogburn M, Turner C and Dahal P 2013 Homomorphic encryption. Proc. Comput. Sci. 20: 502-509

[14] Catalano D I and Visconti I 2007 Hybrid commitments and their applications to zero-knowledge proof systems. Theor. Comput. Sci. 374(1-3): 229-260

[15] Mohan M and Kavitha Devi M K 2017 Privacy preserving non-interactive proof of assets for bitcoin exchanges. Appl. Math. Inf. Sci. 11(3): 749-758

[16] Lindell Y 2015 An efficient transform from Sigma protocols to NIZK with CRS and non-programmable random oracle. In: Proceedings of Theory of Cryptography-12th Theory of Cryptography Conference, TCC 2015, part 1, pp. 93-109

[17] Ciampi M, Persiano G, Sinscalchi L and Visconti I 2016 A transform for NIZK almost as efficient and general as the 
Fiat-Shamir transform without programmable random oracle. In: Proceedings of 13th International Conference TCC 2016-A, LNCS 9563. Beijing, China: Springer, pp. $83-113$

[18] Cramer R, Damgrad I and Schoenmakers B 1994 Proofs of partial knowledge and simplified design of witness hiding protocols. In: Proceedings of CRYPTO94, LNCS 839. Santa Barbara, USA: Springer, pp. 174-187

[19] Garay J A, MacKenzie P and Yang K 2003 Strengthening zero knowledge protocols using signatures. In: Proceedings of EUROCRYPT2003, LNCS 2656. Poland: Springer, pp. 177-194

[20] Canetti R, Goldreich O and Halevi S 2004 The random oracle methodlogy, revisited. J. ACM 51(4): 557-594

[21] Ben-Sasson E, Chiesa A, Genkin D, Tromer E and Virza M 2013 SNARKs for C: verifying program executions succinctly and in zero knowledges. In: Proceedings of CRYPTO, pp. $90-108$

[22] Smith S W 2010 Hardware security modules. London: Chapman and Hall/CRC, pp. 257-278 